\title{
Comparative study of endothelial cell loss in trabeculectomy with combined small incision cataract surgery (SICS), trabeculectomy procedure and temporal small incision cataract surgery (SICS) alone
}

\author{
Sonali Pote ${ }^{1}$, Rupali Maheshgauri ${ }^{2, *}$, R. Magdum ${ }^{3}$, Gira Raninga ${ }^{4}$, Saurabh Ashtamkar ${ }^{5}$ \\ ${ }^{1}$ Junior Resident III, ${ }^{2}$ Associate Professor, ${ }^{3}$ Professor \& HOD, ${ }^{4,5}$ Junior Resident II, Dr. D.Y. Patil Medical College, Pune, \\ Maharashtra, India
}

*Corresponding Author:

Email: rupalim021@gmail.com

\begin{abstract}
When a patient suffers from co-existing cataract and glaucoma, the choice of surgery may be a combined procedure of cataract extraction and trabeculectomy as a one-stage procedure, or trabeculectomy first to restore visual rehabilitation and to prevent progressive optic nerve damage. The main purpose of this study was to evaluate the endothelial cell loss in trabeculectomy with small incision cataract surgery (SICS) against trabeculectomy and SICS alone.

Materials and Methods: This study was conducted on 90 eyes divided into 3 groups among whom 30 cases underwent trabeculectomy through a superior incision, 30 cases underwent SICS with PC-IOL implantation with trabeculectomy through a common superior incision and 30 patients underwent manual SICS with PCIOL.

The corneal endothelial cell count and loss was assessed on first postoperative day, $1^{\text {st }}$ week, $1^{\text {st }}$ month and at 3 months. IOP was assessed on postoperative $1^{\text {st }}$ week, $1^{\text {st }}$ month and at 3 months.

Results: The endothelial cell loss was more in combined SICS with trabeculectomy group than trabeculectomy group alone.

Conclusion: Preoperative evaluation of endothelial cell count must be considered and adequate intra-operative and postoperative measures should be taken to avoid cell loss. However, to avoid further endothelial cell loss due to a two staged surgery, a combined procedure would be a better choice. Combined SICS with trabeculectomy would also reduce the economical burden, postoperative morbidity and the visits of patient to operation theatre.
\end{abstract}

Keywords: Cataract, Endothelial cell loss, Glaucoma, SICS, Trabeculectomy.

\section{Introduction}

In the management of a patient with a cataract with coexisting glaucoma, the options for surgical correction include:

i. A cataract extraction alone in case of phacomorphic glaucoma

ii. Glaucoma filtering procedure alone, followed by cataract removal at a later stage

iii. Combined cataract and glaucoma surgery as a one stage procedure. ${ }^{1,2}$

Combined procedure reduces financial burden as well surgical anxiety of the patients. Patients have less IOP rise post operatively ${ }^{13,14}$ But they have a greater risk of complications such as increased inflammation, hyphaema, hypotony, shallow anterior chamber, and choroidal detachments. For these reasons the operating surgeon should consider each of the basic surgical options and select the approach that seems to be most appropriate for each patient.

The mean endothelial cell count in the normal adult cornea ranges from 2000 to 2500 cells $/ \mathrm{mm}^{2}$, and the count continues to decrease with age. Previous cross sectional studies have shown the normal attrition rate of corneal endothelial cells to be 0.3 to $0.5 \%$ per year. ${ }^{3,4}$ Morphological stability and functional integrity of the corneal endothelium are necessary to maintain long term corneal transparency after cataract surgery. All surgical procedures that involve entry into the anterior chamber damage a proportion of endothelial cells.
There is a wide variation in endothelial cell loss between the various studies even when the mode of surgery is same (e.g., SICS). This is due to various factors like different grades of cataract, different methods of nucleus delivery in SICS, different types of irrigating solution and viscoelastics. ${ }^{7}$

\section{Materials and Methods}

This was a prospective comparative study carried out at a tertiary care hospital in Western Maharashtra from July 2015 to October 2016. All patients were followed up for a period of 3 months postoperatively. Strict compliance with four postoperative visits was followed, the visits being on day $1,1^{\text {st }}$ week, 1 month and $3^{\text {rd }}$ month. 90 patients (selected using consecutive sampling method technique as per the protocol mentioned in data collection) who met the inclusion criteria and reported to the unit during the study period were allocated to 3 different groups as follows:

Group A comprised 30 patients with advanced glaucomatous cupping who underwent trabeculectomy only through a superior incision.

Group B comprised 30 patients of mild to moderate glaucomatous cupping with significant cataract who underwent SICS with posterior chamber intraocular lens (PCIOL) implantation combined with trabeculectomy through a superior incision.

Group C comprised 30 patients of mild to moderate glaucomatous cupping with significant cataract who 
underwent SICS with PCIOL implantation only through a temporal incision.

Ethics Committee clearance was taken before the start of the study.

Informed Consent: Informed written consent was taken on the previous day of the surgery and patients were explained regarding complications of surgery and anaesthesia

\section{Inclusion Criteria:}

1. Primary open angle glaucoma (POAG)

2. Normal tension glaucoma

3. Cataract (except complicated cataract cases) with POAG

\section{Exclusion Criteria:}

1. Patients younger than 18 years old

2. Other ocular diseases besides glaucoma and cataract

3. Significant corneal opacities.

4. Refractive error more than + or -6.00 diopters

5. Patients of angle closure glaucoma.

6. Pseudoexfoliation glaucoma

7. Pigment dispersion glaucoma

Ocular Examination: After doing refraction, best corrected visual acuity was determined. A detailed anterior segment examination on a slit lamp was done. Intraocular pressure was recorded in all cases with the help of Perkin's handheld tonometer. Specular microscopy was done for corneal endothelial cell count. Angle width was measured by gonioscopy and recorded as per Schaffers grading.

In all cases of SICS and combined SICS with trabeculectomy single-piece PMMA intraocular lenses were used.

Preoperative Workup: The refractive power of the cornea was determined by keratometery using Bausch and Lomb keratometer.

1. The axial length of the eye was measured by Ascan ultrasonography.

2. IOL power calculation was done with the help of SRK II formula.

Surgical Technique of Trabeculectomy: Akinesia and anaesthesia was achieved by injection of $8 \mathrm{ml}$ of a mixture of $2 \%$ lignocaine hydrochloride and $0.75 \%$ Bupivicaine in a proportion of $1: 1$, along with $12.5 \mathrm{U} / \mathrm{ml}$ of hyaluronidase given with a sterilized $23 \mathrm{G}(2.54 \mathrm{~cm})$ needle given inferotemporally and superonasally. After the standard cleaning and draping preparation, a wire speculum was applied and fixation of eyeball with superior bridle suture was done. A fornix based conjunctival flap was fashioned and the underlying sclera was exposed. Haemostasis was achieved using wetfield bipolar cautery. A partial thickness limbal based sclera flap of $5 \mathrm{~mm} \times 5 \mathrm{~mm}$ size is reflected down towards the cornea. Excision of trabecular tissue: A narrow strip of the exposed deeper sclera near the cornea containing the canal of Schlemm and the trabecular meshwork was excised. Peripheral iridectomy was performed at 12 O'clock position with Kelly's punch. Closure: the scleral flap was replaced and 10-0 nylon sutures were applied. Then the conjunctival flap was reposited and sutured with interrupted sutures. Sub conjuctival injection of dexamethasone and gentamycin was given. The scleral flap was tested for adequate flow resistance, before closing the conjunctival flap, by injecting balanced salt solution into the anterior chamber via paracentesis.

Surgical Technique of Combined Cataract Extraction and Trabeculectomy: After peribulbar injection of anesthesia, wire speculum was applied, a superior rectus bridle suture is placed. Fornix based conjunctival flap made and haemostasis was achieved with wet field bipolar cautery. A lamellar rectangular shaped scleral flap of $3 \times 4 \mathrm{~mm}$ was made and incorporated into the $7 \mathrm{~mm}$ chord length incision. It was outlined in a square, trapezoidal or half moon shape with a sharp knife. Grooves were made on both sides of the scleral flap along the posterior limbal border to the desired extent of the corneoscleral incision. Sideport entry was made with 15 degree blade. Injection Blurex was injected and AC wash given. An anterior capsulectomy (CCC) was performed. AC entry was made with 3.2 keratome. Hydrodissection was done and nucleus rotated and brought into $\mathrm{AC}$ and delivered. Cortical matter was aspirated using Simcoe's cannula. PC-IOL was implanted into the capsular bag. Triangular scleral flap was excised. Injection Pilocarpine was injected intracamerally. Peripheral iridectomy was done at 12 O'clock position. AC wash was given and 4 interrupted sutures were passed at rectangular flaps ends with 10-0 Ethicon. Ringer's Lactate was injected into anterior chamber via sideport to check patency of bleb. Sideport entry was sealed with hydration. The conjunctival flap was closed with 2 non absorbable sutures. Gentamycin and Dexamethasone were injected subconjunctively. Eyedrop Moxifloxacin was instilled and eyepad given.

Surgical Technique of Small Incision Cataract Extraction with PCIOL Implantation: SICS with intraocular lens implantation was performed through a temporal incision, sutureless self-sealing valvular sclero corneal tunnel incision under local anesthesia. After a peribulbar block, the surgical area was cleansed and draped and the lids were separated using a wire speculum. A fornix-based conjunctival flap was made at the temporal limbus, and bleeders were cauterized with wet field bipolar cautery as required. A $6.0 \mathrm{~mm}$ incision was made on the temporal sclera $1.5 \mathrm{~mm}$ from the limbus. A tunnel was created with a stainless steel crescent knife. A side port was made at 12 or 6 O'clock. Hydroxypropyl methylcellulose (HPMC) 2\% was injected to fill the anterior chamber. A 26-gauge bent capsulotomy needle/forceps was used to create a 
continuous curvilinear capsulorhexis (CCC). An entry was made through the tunnel using a $3.2 \mathrm{~mm}$ keratome. The tunnel was then extended using an extension blade. Hydrodissection was performed using balanced salt solution. The anterior chamber was refilled with viscoelastic (HPMC 2\%) and the nucleus rotated and tumbled into the anterior chamber. The viscoelastic was again injected below and above the nucleus to protect the endothelium. The nucleus was then delivered by viscoexpression. The remaining cortical matter was removed with Simcoe's irrigation/aspiration (I/A) cannula. After which a polymethylmethacrylate posterior chamber IOL was implanted in the capsular bag. The side port entry was sealed with hydration. Conjuctival flaps were reposited and cauterized. A subconjunctival injection $(0.3 \mathrm{~mL}, 10 \mathrm{mg}$ gentamicin and $2 \mathrm{mg}$ dexamethasone) was performed. Eye drop Moxifloxacin $(0.5 \%)$ was instilled and eye pad was given.

Post-Operative Management: All patients were given antibiotic and analgesic-anti inflammatory tablets twice a day for 5 days. Patients were given tapering doses of eyedrop ciprofloxacin and dexamethasone starting with 2 hourly and then tapering off to stop at 6 weeks postoperatively. The cases were examined on day 1, week 1, month 1 and month 3 postoperatively. UCVA, pinhole, was recorded with the help of an illuminated Snellen's chart at a distance of six meters. After doing refraction, BCVA was determined. Anterior segment examination on a slit lamp was performed. Specular microscopy for corneal endothelial cell count was done. The refractive power of the cornea was determined by keratometery. Intraocular pressure was recorded in all cases with the help of Perkin's handheld tonometer. Angle width was measured by gonioscopy and recorded as per Schaffers grading. Direct and indirect ophthalmoscopy was done for fundus evaluation.

\section{Observations and Results}

This was a prospective comparative study conducted on total of 90 eyes. Patients were divided into 3 groups among whom 30 underwent trabeculectomy (Group A), 30 underwent combined SICS with PCIOL with trabeculectomy (Group B) and 30 patients underwent SICS with PCIOL implantation (Group C).

Table 1: Distribution of no. of cases in each study group

\begin{tabular}{|l|c|c|c|}
\hline & $\begin{array}{c}\text { Group } \\
\text { A } \\
\text { (Trab) }\end{array}$ & $\begin{array}{c}\text { Group B } \\
\text { (Combined) }\end{array}$ & $\begin{array}{c}\text { Group C } \\
\text { (Temporal } \\
\text { SICS) }\end{array}$ \\
\hline $\begin{array}{l}\text { No. of } \\
\text { patients } \\
\text { operated }\end{array}$ & $\mathrm{n}=30$ & $\mathrm{n}=30$ & $\mathrm{n}=30$ \\
\hline
\end{tabular}

Table 2: Inter and intra group comparison of IOP $(\mathrm{mmHg})$

\begin{tabular}{|l|c|c|c|c|c|c|}
\hline IOP (mmHg) & Group A & Group B & \multirow{2}{*}{$\begin{array}{c}\text { Group C } \\
(\mathbf{n}=30)\end{array}$} & $(\mathbf{n = 3 0})$ & & \multicolumn{3}{|c|}{ P-value (Inter-Group) } \\
\cline { 5 - 7 } & & & & A v/s B & A v/s C & B v/s C \\
\hline Preop & $33.9 \pm 0.60$ & $33.9 \pm 0.60$ & $15.1 \pm 0.21$ & $0.999^{\mathrm{NS}}$ & $0.001^{* * * *}$ & $0.001^{* * *}$ \\
POW 1 & $15.5 \pm 0.25$ & $16.9 \pm 0.25$ & $16.6 \pm 0.19$ & $0.001^{* * *}$ & $0.003^{* *}$ & $0.948^{\mathrm{NS}}$ \\
POM 1 & $14.3 \pm 0.21$ & $15.0 \pm 0.28$ & $15.0 \pm 0.19$ & $0.080^{\mathrm{NS}}$ & $0.080^{\mathrm{NS}}$ & $0.999^{\mathrm{NS}}$ \\
POM 3 & $13.3 \pm 0.20$ & $12.0 \pm 0.25$ & $13.3 \pm 0.18$ & $0.001^{* * *}$ & $0.999^{\mathrm{NS}}$ & $0.001^{* * *}$ \\
\hline P-value (Intra- & & & & & & \\
Group) & & & & & \\
Preop v Week 1 & $0.001^{* * *}$ & $0.001^{* * *}$ & $0.001^{* * *}$ & & & \\
Preop v Month 1 & $0.001^{* * * *}$ & $0.001^{* * *}$ & $0.662^{\text {NS }}$ & & & \\
Preop v Month 3 & $0.001^{* * *}$ & $0.001^{* * *}$ & $0.001^{* * *}$ & & & \\
\hline
\end{tabular}

Values are Mean \pm Standard error of mean (SEM). Inter-group comparisons are done using one-way analysis of variance (ANOVA) with Bonferroni's PostHoc test for multiple group comparisons. Intra-group comparisons are done using repeated measures analysis of variance (ANOVA) technique. P-value $<0.045$ is considered to be statistically significant. *Pvalue $<0.045, * * \mathrm{P}$-value $<0.01, * * * \mathrm{P}$-value $<0.001$. NS: Statistically Non-Significant. 
Graph 1: Inter and intra group distribution of IOP (mmHg)

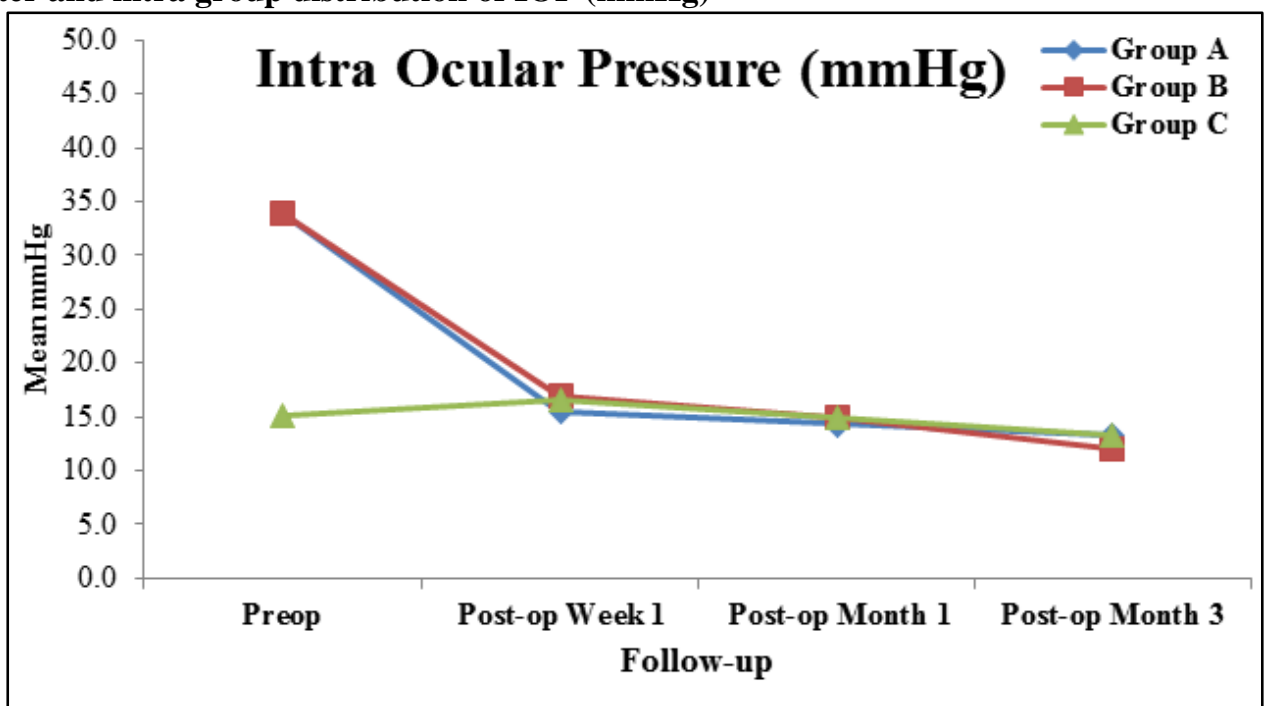

On inter-group comparison of IOP, the mean preoperative IOP did not differ significantly between groups A and B (P-value>0.05). The mean preoperative IOP is significantly higher in groups A and B compared to group $\mathrm{C}$ (P-value $<0.001$ for both). The mean postoperative week 1 IOP is significantly higher in groups $\mathrm{B}$ and $\mathrm{C}$ compared to group A (P-value $<0.01$ for both). The mean post-operative week 1 IOP did not differ significantly between group B and C (P-value>0.05). The mean post-operative month 1 IOP did not differ significantly across three study groups (P-value $>0.05$ for all).The mean post-operative month 3 IOP is significantly higher in groups $\mathrm{A}$ and $\mathrm{C}$ compared to group B (P-value $<0.01$ for both). The mean postoperative month 3 IOP did not differ significantly between group A and C (P-value>0.05).
On intra-group comparison, the mean preoperative IOP in group A is significantly higher compared to post-operative IOP at week1, month 1 and month 3 follow-ups (P-value $<0.001$ for all).In group $\mathrm{B}$, the mean preoperative IOP is significantly higher compared to post-operative IOP at week1, month 1 and month 3 follow-ups (P-value $<0.001$ for all).In Group $\mathrm{C}$, the mean preoperative IOP is significantly higher compared to post-operative IOP at week 1 ,month 1 and month 3 follow-ups (P-value $<0.001$ for all). The mean preoperative IOP did not differ significantly compared to post-operative IOP at month 1 follow-up (Pvalue $>0.05$ )

Graph 2: Inter and intra group comparison of endothelial cell count

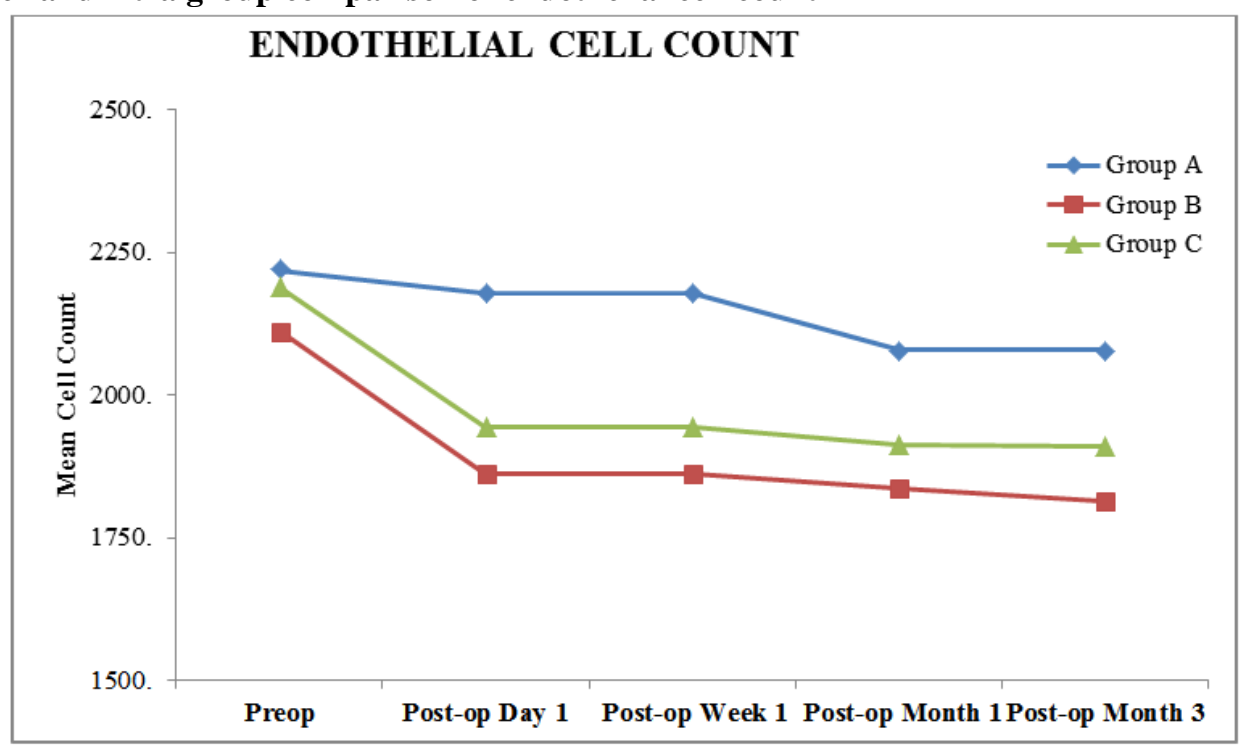


On inter-group comparison, the mean preoperative endothelial cell count did not differ significantly across three study groups $\mathrm{C}$ (P-value>0.05 for all). The mean post-operative day 1 , week 1 , month 1 and month 3 endothelial cell count is significantly higher in group A compared to group B and $\mathrm{C}$ (P-value $<0.001$ for all). The mean post-operative day 1 , week 1 , month 1 and month 3 endothelial cell count did not differ significantly between group $\mathrm{B}$ and $\mathrm{C}$ ( $\mathrm{P}$-value $>0.05$ for all).

On intra-group comparison, the mean preoperative endothelial cell count is significantly higher compared to post-operative endothelial cell count at day 1, week1, month 1 and month 3 follow-ups (P-value $<0.001$ for all).In group $\mathrm{B}$, the mean preoperative endothelial cell count is significantly higher compared to post-operative endothelial cell count at day 1 , week 1 , month 1 and month 3 follow-ups (P-value $<0.001$ for all).In Group C, the mean preoperative endothelial cell count is significantly higher compared to post-operative endothelial cell count at day 1 , week 1 , month 1 and month 3 follow-ups (P-value<0.001 for all).

Table 3: Inter and intra group comparison of endothelial cell loss (\%)

\begin{tabular}{|c|c|c|c|c|c|c|}
\hline \multirow[t]{2}{*}{ Cell Loss (\%) } & \multirow{2}{*}{$\begin{array}{c}\text { Group A } \\
(\mathbf{n}=\mathbf{3 0})\end{array}$} & \multirow{2}{*}{$\begin{array}{c}\text { Group B } \\
(\mathbf{n}=\mathbf{3 0})\end{array}$} & \multirow{2}{*}{$\begin{array}{c}\text { Group C } \\
(n=30)\end{array}$} & \multicolumn{3}{|c|}{ P-value (Inter-Group) } \\
\hline & & & & A v/s B & A v/s C & $\mathrm{B}$ v/s C \\
\hline Preop & 0 & 0 & 0 & -- & -- & -- \\
\hline POD 1 & 1.9 & 11.9 & 11.2 & $0.001^{\text {**** }}$ & $0.001^{\text {**** }}$ & $0.039^{*}$ \\
\hline POW 1 & 1.9 & 11.9 & 11.2 & $0.001^{* * * *}$ & $0.001^{\text {**** }}$ & $0.039^{*}$ \\
\hline POM 1 & 6.3 & 13.3 & 12.7 & $0.001^{\text {**** }}$ & $0.001^{* * *}$ & $0.042^{*}$ \\
\hline POM 3 & 6.3 & 14.1 & 12.8 & $0.001^{\text {**** }}$ & $0.001^{\text {**** }}$ & $0.033^{*}$ \\
\hline $\begin{array}{l}\text { P-value (Intra- } \\
\text { Group) }\end{array}$ & & & & & & \\
\hline Preop v Day 1 & $0.001^{* * * *}$ & $0.001^{* * * *}$ & $0.001^{\text {*** }}$ & & & \\
\hline Preop v Week 1 & $0.001^{* * * *}$ & $0.001^{* * *}$ & $0.001^{\text {**** }}$ & & & \\
\hline Preop v Month 1 & $0.001^{* * * *}$ & $0.001^{* * * *}$ & $0.001^{* * *}$ & & & \\
\hline Preop v Month 3 & $0.001^{* * * *}$ & $0.001^{* * *}$ & $0.001^{* * *}$ & & & \\
\hline
\end{tabular}

Values are Mean \pm Standard error of mean (SEM). Inter-group comparisons are done using one-way analysis of variance (ANOVA) with Bonferroni's PostHoc test for multiple group comparisons. Intra-group comparisons are done using repeated measures analysis of variance (ANOVA) technique. P-value $<0.045$ is considered to be statistically significant. $* \mathrm{P}$ value $<0.045$, **P-value $<0.01, * * * \mathrm{P}$-value $<0.001 . \mathrm{NS}$ : Statistically Non-Significant.

\section{Graph 3: Inter and intra group comparison of endothelial cell loss (\%)}

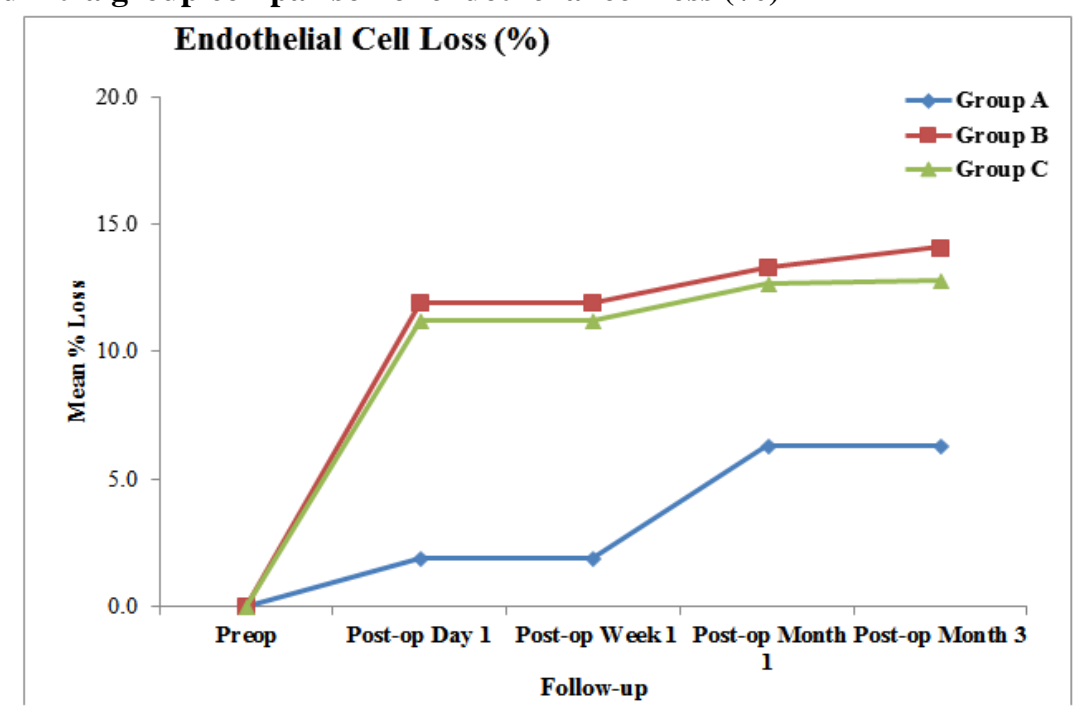

On inter-group comparison, the mean postoperative day1, week1, month 1 and month 3, percentage cell loss is significantly higher in group $\mathrm{B}$ and $\mathrm{C}$ compared to group $\mathrm{A}$ ( $\mathrm{P}$-value $<0.05$ for all). The mean post-operative day1, week 1 , month 1 and month 3 , percentage cell loss is significantly higher in groups $\mathrm{B}$ compared to group $\mathrm{C}(\mathrm{P}$-value $<0.05$ for all $)$. 
On intra-group comparison, in group $\mathrm{A}$, the mean post-operative cell loss at day1, week1, month 1 and month 3 follow-ups is significantly higher compared to the preoperative endothelial cell count $(\mathrm{P}$-value $<0.001$ for all). In Group B, the mean post-operative cell loss at day1, week1, month 1 and month 3 follow-ups is significantly higher compared to the preoperative endothelial cell count (P-value $<0.001$ for all). In Group $\mathrm{C}$, the mean post-operative cell loss at day1, week1, month 1 and month 3 follow-ups is significantly higher compared to the preoperative endothelial cell count ( $\mathrm{P}$ value $<0.001$ for all).

\section{Discussion}

In the past few decades cataract surgery has undergone drastic changes. Rapid advancement in surgical methods allows safe wound closure with minimum complications. Attention has thus shifted from anatomical to optical results. Cataract surgery is now becoming a form of refractive surgery.

It is a common experience as an ophthalmologist to come across various vision related problems in patients who have undergone uncomplicated cataract surgery with PCIOL implantation.

This study was conducted on 90 eyes divided into 3 groups among whom 30 cases underwent trabeculectomy through a superior incision, 30 cases underwent SICS with PCIOL implantation with trabeculectomy through a common superior incision and 30 cases underwent SICS with PCIOL implantation through a temporal incision.

The corneal endothelial cell count and loss was assessed on first postoperative day, week 1 , month 1 and month 3 . While IOP was assessed on postoperative week 1 , month 1 and month 3 and angle width was assessed on postoperative month 1 and month 3 .

Endothelial Cell Count and Loss: The SICS technique carries greater risk of endothelial loss that is mainly attributed to surgical manipulation in the anterior chamber close to corneal endothelium and endothelial trauma during the nucleus delivery from the anterior chamber. The manipulation during nucleus delivery further increases by the temporal scleral tunnel approach used in this study (scleral tunnel approach was performed to reduce the postoperative astigmatism as well preserve the superior space for future Trabeculectomy if requires). Various modifications of SICS (irrigating vectis, viscoexpression of the nucleus, anterior chamber maintainer, high density viscoelastics) have significantly reduced the endothelial cell loss

On inter-group comparison, the average preoperative endothelial cell count did not differ significantly across three study groups ( $\mathrm{P}$-value $>0.05$ for all). The average post-operative day 1 , week 1 , month 1 and month 3 endothelial cell count is significantly higher in group A compared to groups B and $\mathrm{C}$ (P-value $<0.001$ for all). The average postoperative day 1 , week 1 , month 1 and month 3 endothelial cell count did not differ significantly between groups B and C (P-value $>0.05$ for all). It is accepted all over the world that there is definite loss of some endothelial cells with all techniques of cataract surgery. ${ }^{8}$

On intra-group comparison, in Group A, the average preoperative endothelial cell count is significantly higher compared to post-operative endothelial cell count at day 1 , week 1 , month 1 and month 3 follow-ups (P-value $<0.001$ for all).

A study by Arnavielle $\mathrm{S}$ et al conducted corneal endothelial cell changes after trabeculectomy in 2007 in France concluded that cell loss was 7\% after trabeculectomy. ${ }^{5}$

In Group B, the average preoperative endothelial cell count is significantly higher compared to postoperative endothelial cell count at day 1 , week1, month 1 and month 3 follow-ups (P-value $<0.001$ for all). In Group $\mathrm{C}$, the average preoperative endothelial cell count is significantly higher compared to post-operative endothelial cell count at day 1 , week1, month 1 and month 3 follow-ups (P-value $<0.001$ for all).

María I et al conducted a study in Spain in 2009 on endothelial cell damage after glaucoma surgery and combined glaucoma and cataract surgery in one or two steps using confocal biomicroscopy and concluded that combined trabeculectomy, phacoemulsification and intraocular lens implantation causes more endothelial cell damage than trabeculectomy alone, and the twostep combined procedure causes more damage to the endothelium than the one-step combined procedure. ${ }^{9}$

On inter-group comparison, the average postoperative day1, week1, month 1 and month 3 endothelial cell loss is significantly higher in groups B and $\mathrm{C}$ compared to group $\mathrm{A}$ ( $\mathrm{P}$-value $<0.05$ for all). The average post-operative day1, week1, month 1 and month 3 endothelial cell loss is significantly higher in groups B compared to group C (P-value $<0.05$ for all).

On intra-group comparison, in Group A, the postoperative endothelial cell loss at day 1 , week 1 , month 1 and month 3 follow-ups is significantly higher compared to preoperative endothelial cell count (Pvalue $<0.001$ for all). We have found that trabeculectomy alone causes the loss of $6.3 \%$ of the endothelial cells. In prospective studies, Arnavielle $S$ et al and Soro-Martínez MI et al and have found a similar percentage of cell loss after trabeculectomy. ${ }^{5,6}$ There are many variations in the trabeculectomy technique, and it may be possible that the percentage of cell loss that we find is due to the fact that we perform a macrotrabeculectomy technique, because nonpenetrating glaucoma surgery has been shown to cause lesser cell loss than penetrating surgery. ${ }^{5}$

In Group B, the post-operative endothelial cell loss day 1 , week1, month 1 and month 3 follow-ups is significantly higher compared to preoperative endothelial cell count (P-value $<0.001$ for all). 
In Group C, the post-operative endothelial cell loss at day 1 , week 1 , month 1 and month 3 follow-ups is significantly higher compared to preoperative endothelial cell count (P-value $<0.001$ for all). Our study had a similar endothelial cell loss following SICS as a study done by Gogate et al in February 2010 and by Ganekal $\mathrm{S}$ et al in $2014 .^{10,11}$

Endothelial cell loss is more likely related to corneal endothelial cell remodeling after the trauma of surgery than to ongoing age related cell loss. ${ }^{12}$ Additionally, we used 3 months follow up to reduce the number of patients lost to follow up, which increases the validity of the present study. SICS is still a safe and cost effective option in the developing world. Proper case selection, diligent surgery, and adequate postoperative care are essential to maintain a clear cornea.

\section{Conclusion}

Significant endothelial cell loss was noted in all three groups. The endothelial cell loss was more in combined SICS with trabeculectomy group than SICS with PCIOL implantation group and trabeculectomy group alone. Thus preoperative evaluation of endothelial cell must be considered and adequate intraoperative and postoperative measures should be taken. However, to avoid further endothelial cell loss due to a two staged surgery, a combined procedure would be a better choice. Combined SICS with trabeculectomy would also reduce the economical burden, postoperative morbidity and the visits of patient to operation theatre.

There is a further scope to have a study on comparative analysis of endothelial cell loss following trabeculectomy followed by temporal incision SICS as a two stage procedure and a combined SICS with trabeculectomy as one stage procedure.

\section{References}

1. Duke-Elder S. System of Ophthalmology, Vol 5, Ophthalmic optics and refraction, St. Louis, 1970, CV Mosby.

2. Krupin, T, Feitl, ME, Bishop, KI. Postoperative intraocular pressure rise in open- angle glaucoma patients after cataract or combined cataract filteration surgery. Ophthalmology 1989;96:579.

3. Yee RW, Matsuda M, Schultz RO, Edelhauser HF. Changes in the normal corneal endothelial cellular pattern as a function of age. Curr Eye Res 1985;4:671-8.

4. Carlson KH, Bourne WM, McLaren JW, Brubaker RF. Variations in human corneal endothelial cell morphology and permeability to fluorescein with age. Exp Eye Res 1988;47:27-41.

5. Arnavielle S, Lafontaine PO, Bidot S, Creuzot-Garcher C, D'Athis P, Bron AM. Corneal endothelial cell changes after trabeculectomy and deep sclerectomy. J Glaucoma. 2007 May;16(3):324-8.

6. George R, Rupauliha P, Sripriya AV, Rajesh PS, Vahan PV, Praveen S. Comparison of endothelial cell loss and surgically induced astigmatism following conventional extracapsular cataract surgery, manual small-incision surgery and phacoemulsification. Ophthalmic Epidemiol 2005;12:293-7.

7. Mencucci R, Ponchietti C, Virgili G, Giansanti F, Menchini U. Corneal endothelial damage after cataract surgery: Microincision versus standard technique. $J$ Cataract Refract Surg 2006;32:1351-4.

8. Helga PS, Castro D, Fernandez LE, David TV, Kerry DS. Randomized double-masked clinical trial evaluating corneal endothelial cell loss after cataract extraction and intraocular lens implantation: fluid-based system versus ultrasound Phacoemulsification. Cornea 2006;25:1043-5.

9. Soro-Martínez MI, Villegas-Pérez MP, Sobrado-Calvo P, Ruiz-Gómez JM, Miralles de Imperial Mora-Figueroa J. Corneal endothelial cell loss after trabeculectomy or after phacoemulsification, IOL implantation and trabeculectomy in 1 or 2 steps. Graefes Arch Clin Exp Ophthalmol. $2010 \mathrm{Feb}$;248(2):249-56.

10. Gogate P, Ambardekar P, Kulkarni S, et al. Comparison of endothelial cell loss after cataract surgery: phacoemulsification versus manual small-incision cataract surgery: six-week results of a randomized control trial. J Cataract Refract Surg. 2010 Feb;36(2):247-53.

11. Ganekal S, Nagarajappa A. Comparison of Morphological and Functional Endothelial Cell Changes after Cataract Surgery: Phacoemulsification versus Manual Small-Incision Cataract Surgery. Middle East African Journal of Ophthalmology. 2014;21(1):56-60.

12. Edelhauser HF, Sanders DR, Azar R, Lamielle H. ICL in Treatment of Myopia Study Group. Corneal endothelial assessment after ICL implantation. J Cataract Refract Surg 2004;30:576-83.

13. Duke-Elder S. System of Ophthalmology, Vol 5, Ophthalmic optics and refraction, St. Louis, 1970, CV Mosby.

14. Krupin, T, Feitl, ME, Bishop, KI. Postoperative intraocular pressure rise in open- angle glaucoma patients after cataract or combined cataract filteration surgery. Ophthalmology 1989;96:579. 